\title{
COMMUTATIVITY AND COMMON FIXED POINTS IN RECURSION THEORY
}

\author{
JAMES C. OWINGS, JR. ${ }^{1}$
}

Let $N$ be the set of nonnegative integers and, for $e \in N$, let $\phi_{\theta}$ be the partial recursive function of one argument having index $e$. In 1938 [1, The Recursion Theorem] Kleene showed that if $f$ is any recursive function then, for some number $c, \phi_{c} \simeq \phi_{f(c)}$. It follows that if $W_{e}$ (the recursively enumerable (r.e.) set with index $e$ ) is defined as the domain of $\phi_{e}$, then $W_{c}=W_{f(c)}$. Call a number-theoretic function $h$ well-defined on the r.e. sets if, for all $m, n \in N, W_{m}=W_{n} \rightarrow W_{h(m)}$ $=W_{h(n)}$. In this paper we show that if $f, g$ are recursive functions which are well-defined on the r.e. sets and which commute as maps of the r.e. sets (i.e., for all $\left.n \in N, W_{f(o(n))}=W_{g(f(n))}\right)$, then they have a common fixed point (i.e., for some $\left.e \in N, W_{\theta}=W_{f(e)}=W_{o(e)}\right)$. We also give an example which shows that the assumption of well-definedness cannot be eliminated.

First we prove a lemma related to the Myhill-Shepherdson Theorem [2, p. 359, Theorem XXIX (6)]. From now on, whenever $f$ is well defined on the r.e. sets and $W$ is an r.e. set, we shall write $f(W)$ for $W_{f(e)}$ where $e$ is any number such that $W=W_{e}$.

Leмma. If $f$ is a partial recursive function well-defined on the r.e. sets and $W$ is an r.e. set, then

$$
f(W)=\bigcup\{f(F) \mid F \subseteq W \& F \text { is finite }\} .
$$

Proof. Our proof consists of two applications of Kleene's Recursion Theorem.

$f(W) \subseteq \bigcup\{f(F) \mid F \subseteq W\}$ : Suppose $n \in f(W)$. Let $h$ be a recursive function such that, for all $x$,

$$
\begin{aligned}
W_{h(x)} & =W \quad \text { if } n \in f\left(W_{x}\right), \\
& =\text { some finite subset } F \text { of } W \text { if } n \in f\left(W_{x}\right),
\end{aligned}
$$

and choose $c$ satisf ying $W_{c}=W_{h(c)}$. Clearly, $W_{c} \subseteq W$. Suppose $n \in f\left(W_{c}\right)$. Then $W_{c}=W$, a contradiction, since $n \in f(W)$. So $n \in f\left(W_{c}\right)$. But then $W_{c}$ is finite, so $n \in \bigcup\{f(F) \mid F \subseteq W\}$.

$\bigcup\{f(F) \mid F \subseteq W\} \subseteq f(W)$ : Suppose $F \subseteq W$ and $n \in f(F)$. Let $h$ be a recursive function such that, for all $x$,

Received by the editors March 21, 1969.

1 Partially supported by NSF Grant GP-6897. 


$$
\begin{aligned}
W_{k(x)} & =F & & \text { if } n \in f\left(W_{x}\right), \\
& =W & & \text { if } n \in f\left(W_{x}\right),
\end{aligned}
$$

and choose $c$ satisfying $W_{c}=W_{h(c)}$. Suppose $n \notin f\left(W_{c}\right)$. Then $W_{c}=F$, a contradiction, since $n \in f(F)$. So $n \in f\left(W_{c}\right)$. But then $W_{c}=W$, so $n \in f(W)$.

THEOREM. Let $f, g$ be recursive functions such that

$$
W_{m}=W_{n} \rightarrow\left(W_{f(m)}=W_{f(n)} \& W_{\varrho(m)}=W_{\varrho(n)} \& W_{f(\vartheta(m))}=W_{\varrho(f(n)))} .\right.
$$

Let $V=U_{k>0}(f \circ g)^{k}(\varnothing)$. Then $V$ is r.e., $V=f(V)=g(V)$ and, for all $V^{\prime}$, if $V^{\prime}=f\left(V^{\prime}\right)=g\left(V^{\prime}\right)$, then $V^{\prime} \supseteq V$.

Proof. Let $V_{0}=\varnothing, V_{n+1}=f\left(g\left(V_{n}\right)\right)$. Since $W_{0}=\varnothing$, if we define $k(0)=0 ; k(n+1)=f(g(k(n)))$, then $V_{n}=W_{k(n)}$ for all $n \geqq 0$. So

$$
V=\bigcup_{n>0} V_{n}=\bigcup_{n>0} W_{k(n)}
$$

is an r.e. set.

If $h$ is well defined on the r.e. sets and $W, W^{\prime}$ are r.e. sets with $W \subseteq W^{\prime}$, then, by the lemma,

$$
h(W)=U h(F)(F \subseteq W) \subseteq U h(F)\left(F \subseteq W^{\prime}\right)=h\left(W^{\prime}\right) .
$$

We have $\varnothing \subseteq f(\varnothing), \varnothing \subseteq g(\varnothing)$. So

$$
g(\varnothing) \subseteq g(f(\varnothing))=f(g(\varnothing)), \quad f(\varnothing) \subseteq f(g(\varnothing)) .
$$

Hence $V_{0} \subseteq f\left(V_{0}\right) \subseteq V_{1}, \quad V_{0} \subseteq g\left(V_{0}\right) \subseteq V_{1}$. Inductively, assume $V_{n}$ $\subseteq g\left(V_{n}\right) \subseteq V_{n+1}, V_{n} \subseteq f\left(V_{n}\right) \subseteq V_{n+1}$. Then

$$
\begin{aligned}
& V_{n+1}=f\left(g\left(V_{n}\right)\right) \subseteq f\left(V_{n+1}\right), \\
& V_{n+1}=g\left(f\left(V_{n}\right)\right) \subseteq g\left(V_{n+1}\right),
\end{aligned}
$$

so that

$$
g\left(V_{n+1}\right) \subseteq g\left(f\left(V_{n+1}\right)\right)=f\left(g\left(V_{n+1}\right)\right)=V_{n+2}
$$

and

$$
f\left(V_{n+1}\right) \subseteq f\left(g\left(V_{n+1}\right)\right)=V_{n+2} .
$$

Thus

$$
V_{n+1} \subseteq f\left(V_{n+1}\right) \subseteq V_{n+2}, \quad V_{n+1} \subseteq g\left(V_{n+1}\right) \subseteq V_{n+2} .
$$

So, for all $n \geq 0, V_{n} \subseteq f\left(V_{n}\right) \subseteq V_{n+1}, V_{n} \subseteq g\left(V_{n}\right) \subseteq V_{n+1}$.

This gives

$$
V=\bigcup_{n>0} V_{n}=\bigcup_{n>0} f\left(V_{n}\right)=\bigcup_{n>0} g\left(V_{n}\right)
$$


So

$$
f(V)=\bigcup f(F)(F \subseteq V)=\bigcup_{n>0}\left(\bigcup f(F)\left(F \subseteq V_{n}\right)\right)=\bigcup_{n>0} f\left(V_{n}\right)=V,
$$

and

$$
g(V)=\bigcup g(F)(F \subseteq V)=\bigcup_{n>0}\left(\bigcup g(F)\left(F \subseteq V_{n}\right)\right)=\bigcup_{n>0} g\left(V_{n}\right)=V .
$$

Also, $V$ is the least common fixed point. For let $V^{\prime}$ be any other common fixed point. Trivially $V_{0}=\varnothing \subseteq V^{\prime}$; suppose $V_{n} \subseteq V^{\prime}$. Then $g\left(V_{n}\right) \subseteq g\left(V^{\prime}\right)=V^{\prime}$, so that

$$
V_{n+1}=f\left(g\left(V_{n}\right)\right) \subseteq f\left(V^{\prime}\right)=V^{\prime} .
$$

Hence $V=U_{n>0} V_{n} \subseteq V^{\prime}$.

The reader will detect a close connection between the above proof and Kleene's proof of his "first" recursion theorem [1, p. 66].

There is a version of Theorem 2 for partial recursive functions, rather than r.e. sets. One can replace $W$ by $\phi,=$ by $\simeq$, $U$ by "least common extension of" and $\supseteq$ by "extends."

We note the assumption of well-definedness in Theorem 2 is necessary. For let $e_{n}$ be the Gödel number of the finite system of equations $h(\boldsymbol{n})=\boldsymbol{n}$ where $h$ is a function letter and $\boldsymbol{n}$ is the numeral for $n$. Then, for all $n, W_{e_{n}}=\{n\}$. Define

$$
\begin{aligned}
& f(x)=\mu y\left(y \geqq x \&(\exists n)\left(y=e_{n}\right)\right), \\
& g(x)=\mu y\left(y \geqq x \&(\exists n)\left(x \leqq e_{n} \& y=e_{n+1}\right)\right) .
\end{aligned}
$$

Then $f(g(x))=g(f(x))=g(x)$, so $f$ and $g$ commute as number-theoretic functions. $f$ and $g$ are recursive, but for all $x, W_{f(x)} \neq W_{o(x)}$. Thus $f$ and $g$ cannot possibly have a common fixed point.

\section{REFERENCES}

1. S. C. Kleene, Introduction to metamathematics, Van Nostrand, Princeton, N. J., 1952. MR 14, 525.

2. Hartley Rogers, Jr., Theory of recursive functions and effective computability, McGraw-Hill, New York, 1967. MR 37 \#61.

UNIVERSITY OF MARYLAND 